\title{
Influence of the Service Delivery Environment on Family Planning Outcomes in Nigeria
}

\author{
Anastasia J. Gage ${ }^{1}$ and Delayo L. Zomahoun ${ }^{2}$ \\ ${ }^{1}$ Department of Global Community Health and Behavioral Sciences, Tulane University School of \\ Public Health and Tropical Medicine, New Orleans, LA 70112, \\ agage@tulane.edu; agage11961@aol.com \\ ${ }^{2} 2110$ Clairmeade Valley Road NE, Atlanta, GA 30329, \\ laurelzom@gmail.com
}

\begin{abstract}
Using linked data from a 2009 health facility and household survey and multilevel logit models, this paper examined the association of the service delivery environment with contraceptive outcomes in Nigeria. The findings showed significant positive associations of the number of modern contraceptive methods provided and quality assurance systems used by health facilities in the Local Government Area (LGA) with the odds of knowing three or more modern methods. These odds were significantly higher among rural than urban residents. The LGA indices of provider training in FP and provider-client interaction had significant positive associations, while a perceived need for more staff in FP health facilities had a significant negative association with the odds of current use of a modern method. The positive association between the index of FP provider-client interaction and current use of a modern method was significantly stronger among women than men and in semi-urban than urban areas.
\end{abstract}

Keywords: family planning, method mix, quality of care, service delivery, Nigeria

\section{Introduction}

In 2013 , only $10 \%$ of currently married women in Nigeria were using a modern method of contraception. Although unmet need for spacing declined from 2008 to 2013 (Austin, 2015), 16\% of married Nigerian women who wanted to avoid pregnancy were not using safe and effective family planning (FP) methods (National Population Commission and ICF International, 20I4). The low level of contraceptive uptake in Nigeria has been attributed to numerous factors including religious and cultural beliefs, fear of side effects and associated health concerns, and misconceptions (AsekunOlarinmoye et al., 2013, Tsui et al., 201I). Some authors have noted that the service delivery environment in Nigeria is characterized by inadequate human resources for health, inadequate funding, commodity stock outs, limited contraceptive availability, inadequate infrastructure, and poor staff remuneration (Charyeva et al., 2015, Levy et al., 2014, Uneke et al., 2014). Although there is increased recognition of the importance of FP service quality for improving contraceptive behavior (Bruce, 1990, Campbell et al., 2015, Jain, 1989, Ross and Stover, 2013, Tessema et al., 2016, Tumlinson et al., 2015), the influence of the service delivery environment on contraceptive outcomes is not well understood.
Bruce's (1990) framework on fundamental elements of the quality of care has provided a reference for measuring the readiness of health facilities (HFs) to provide FP services and the quality of care received by clients in provider-client contacts. According to the framework, quality comprises six elements:

(I) choice of methods;

(2) information given to users,

(3) technical competence;

(4) interpersonal relations;

(5) follow-up or continuity mechanisms;

(6) appropriate constellation of services.

Choice of methods refers to both the number of contraceptive methods offered regularly and the extent to which methods offered meet the needs of significant subgroups. Information given to clients refers to information imparted during provider-client interactions that enables clients' informed choice and satisfaction. Technical competence refers to factors such as clinical techniques, observance of protocols, and maintenance of aseptic conditions required to provide IUDs, implants, and sterilization. Interpersonal relations refer to the personal dimensions of service, which Bruce (1990) described as including the program's mission and ideology, management style, resource allocation, the ratio of workers to clients, and supervisory structure. Mechanisms to encourage continuity are described as 
including but not limited to community media, processes for forwarding appointments, and home visits by health workers (HWs). Appropriate constellation of services refers to the situating of FP services so that they are convenient and acceptable to clients, which may happen in vertical programs or in the context of maternal and child health, postpartum, or comprehensive reproductive health $(\mathrm{RH})$ services.

Efforts to relate the quality of care to contraceptive behavior have been characterized by marked differences in definitions of quality, outcomes, client populations examined, levels of analysis (Koenig et al., 1997), and lack of data and standardized criteria for assessing the quality of service provision (Campbell et al., 2015). Although the evidence is inconsistent, effects often small in magnitude, and causality a concern, a number of studies have found significant associations between elements of the FP services delivery environment and contraceptive outcomes, even after controlling for individual-level factors. HF infrastructure and equipment (Do and Koenig, 2007), the range of methods available (Jain, 1989, Magnani et al., 1999, Steele et al., 1999), and the presence of trained providers (Ali, 200I) have been found to be positively associated with contraceptive use. However, other studies have found a weak or non-significant link between service delivery characteristics and contraceptive behavior (Baumgartner et al., 20I2).

Few studies have explored how the actual quality of provider-client interaction is related to contraceptive use or discontinuation. A study by Arends-Kuenning and Kessy (2007) found that, in Tanzania, information given to clients and technical competence had significant positive associations with contraceptive use, but interpersonal relations (measured by how comfortable the provider was in discussing sexual behavior related to sexuallytransmitted infection (STI)/Human Immune Deficiency Virus (HIV) with clients and if the provider routinely asked the patient any questions if an STI was suspected) did not. A recent study by Tumlinson et al. (2015) found that asking clients about their preferences, providing assistance with method selection and information on side effects, and provider treatment of clients were all associated with a significantly increased likelihood of current modern contraceptive use, especially among younger and less educated women in urban Kenya.

Despite substantial investment of effort and resources to reduce its population growth rate to two percent or lower by 2015 and its total fertility rate by at least 0.6 children ever five years, Nigeria has been unable to meet its policy targets (National Population Commission and Health Policy Project,
20I5), and is projected to become the third largest nation in the world by mid-century (United Nations, 2015). Analyzing how demand- and supply- side factors influence the utilization of FP products and services is a fundamental first step in devising strategies to meet national population policy objectives. This study attempts to contribute to these efforts by examining the association between the FP service delivery environment and contraceptive knowledge and use in four states and the Federal Capital Territory (FCT) of Nigeria.

\section{Data and Methods}

Data were derived from the 2009 end-of project survey for the Community Participation for Action in the Social Sectors (COMPASS) project in Nigeria. Household, HF and school surveys were implemented by MEASURE Evaluation/Tulane University and the Center for Research, Evaluation and Resource Development in 5I local government areas (LGAs) in the FCT and the states of Bauchi, Kano, Lagos, and Nasarawa where the COMPASS project was implemented. The purpose of COMPASS was to integrate health and education by enhancing $\mathrm{FP} / \mathrm{RH}$ services, promoting child survival and improving basic literacy and numeracy. The household survey used a multi-stage stratified sampling design and collected information on $\mathrm{RH}$, child health, and primary school education among a representative sample of women aged 15-49 and men aged 15-64. At the first stage of sampling, enumeration areas (EAs) were selected within each state, with probability proportional to the number of LGAs per state as follows: I:I:2:2:I for Bauchi, FCT, Kano, Lagos, and Nasarawa, respectively. At the second stage of sampling, 25 households were selected within each sample EA using systematic random sampling.

The survey of primary health care (PHC) facilities (comprehensive health care centers, public PHC centers, health clinics, maternity clinics, private clinics, uniformed services clinics, health posts, and dispensaries) and patent medicine vendors (PMVs)) was implemented at the same time as the household survey. The sample for the HF survey was drawn from a list of all public and private PHCs and PMVs serving sampled households and as a result, included some service delivery points that were located outside of EAs selected for the household survey. Due to sample size considerations, the LGA (as opposed to the EA) was used to link the facility and household survey in order to determine the influence of health service characteristics on individual health outcomes. To the extent possible, all PHC facilities and PMVs were included when defining LGA-based measures of the service delivery environment. 
The Institutional Review Board of Tulane University and the COMPASS Institutional Review Committee granted ethical approval for the study. Written and witnessed consent were obtained from respondents prior to interview. Eighty-eight percent of selected HFs and $99 \%$ of selected household respondents were completely interviewed.

\section{Measures}

Outcomes

Two binary outcomes were examined:

(a) Knowledge (spontaneous) of three or more modern methods of contraception (i.e., female sterilization, male sterilization, pill, intrauterine device (IUD), injection, implants, male condom, female condom, diaphragm, foam/jelly, or emergency contraception (EC)).

(b) Current use of a modern method (i.e., use of any of the afore-mentioned methods except EC).

LGA-level variables

LGA-level variables included:

Mean number of FP methods provided: All FP HFs were asked whether they provided the following contraceptive methods:

(I) combined oral contraceptive pill,

(2) progesterone-only pill,

(3) IUD,

(4) injectable Depo Provera,

(5) injectable Noristerat,

(6) implant (e.g., Norplant),

(7) diaphragm/cap,

(8) male condom,

(9) female condom,

I0) foaming tablets/spermicides,

(I I) EC;

(I2) counseling about natural FP; and

(13) counseling about dual protection (prevention of pregnancy and STI/HIV). We calculated the total number of methods provided by each FP HF and averaged that over all FP HFs in the LGA. The mean ranged from 0 to $\mathrm{II}$ and was a proxy for method choice potential in the LGA.

Three variables measured the availability of systems that supported quality FP service delivery:

Mean number of quality assurance (QA) methods used by FP HFs: An additive index was first created at the HF-level to measure how many of the following QA methods were used routinely: supervisory checklist for health system components (e.g. servicespecific equipment, medication, and records) based on standards and protocol; supervisory checklist for health service provision (e.g. observation checklist) based on standards and protocol; system for identifying and addressing quality of care that was implemented by staff or specific service level (e.g. not carried out facility wide); facility-wide review of mortality; periodic audit of medical records or service registers; QA committee/team; and Regional/District Health Management Teams. The alpha coefficient for the variables comprising the seven-item QA index was 0.933 . The index was then averaged over FP non-PMVs in the LGA and ranged from 0 to 6.

High FP supervision: Principal components analysis (PCA) was first used to create an index that captured the content of FP supervisory visits in the past 6 months. The index was based on two questions:

(I) "When was the last time a supervisor from outside this facility visited the facility?"

(2) If a supervisory visit was received in the last 6 months, then the following question was asked: The last time that a supervisor from outside the facility visited, did the supervisor:

a) Check some registers/books?

b) Discuss problems?

c) Discuss policy/administrative issues?

d) Discuss technical protocols/practices/issues?

e) Hold an official staff meeting?

f) Observe individual staff providing services;

g) Meet with staff individually?

h) Meet with clients?

i) Do anything else?".

Binary variables indicated the presence/absence in the LGA of a FP non-PMV that received the aforementioned components of supervision. Cronbach's alpha coefficient for these LGA-level variables was 0.924. For the PCA, we omitted the component pertaining to "check some registers/books" due to multicollinearity. The Kaiser-Meyer-Olkin (KMO) measure of sampling adequacy, which takes on values between 0 and $\mathrm{I}$, with small values indicating that overall the variables have too little in common to warrant a PCA, was 0.859 . As the eigen value of the first component explained $70 \%$ of the variation in the other components, its predicted value was used to create the LGA-based FP supervision index. We differentiated LGAs that were at/above the median value of the resulting index (high) from those that were not (low).

Mean number of methods used by FP HFs to solicit client opinion: This variable, which pertained to FP non-PMVs in the LGA, captured the extent to which HFs collected data revealing the opinion of clients on issues related to their satisfaction and was derived from the following questions:

(I) Does this facility have any system for determining client opinion about the HF or services? (2) Which of 
the following systems does the facility have for determining client opinion:
(a) Suggestion box?
(b) Client survey form?
(c) Client interview?
(d) Other system?

We first calculated the number of methods used by the HF to determine client opinion and averaged that over all FP non-PMVs in the LGA. The index ranged from 0 to 2 and was a reflection of the general service provision environment.

Index of provider training in FP: PCA was used to create an 8-item index measuring the availability in the LGA of FP providers who had received lifetime in-service training in the following areas of service provision: FP counseling; IUD insertion; minilaparotomy; no-scalpel vasectomy; implants (e.g., Norplant); STI syndromic management; STI diagnosis and treatment; and post-abortion care. Each item was dichotomous. The KMO coefficient was 0.860 . The first component explained $61 \%$ of the variation in the other components and its predicted values were used to derive the index, which ranged from 3.356 to 2.459 .

Availability of basic resources for FP examination: This binary variable measured whether any HFs in the LGA were prepared to provide FP services at a minimum. The LGA was assigned the value "I" if any of its HFs had all of the following items deemed essential for the provision of FP services: equipment for examination (a spotlight source (flashlight or examination light); examination bed/table); items for infection control, which included the following:

(i) hand washing items (soap and towel);

(ii) water for hand washing;

(iii) clean and sterilized gloves;

(iv) decontamination solution for clinical equipment; and

(v) sharps box.

We also examined the availability of other equipment:
a) blood pressure gauge;
b) stethoscope;
C) weighing scale; and
d) sterile needle and syringe.

Mean number of items to support FP counseling: Questions inquired as to whether visual aids were available in the counseling or service provision area for teaching about:

(I) FP methods,

(2) STIs,

(3) HIV/AIDS;

(4) use of male condoms, or

(5) breast self-exams.
Questions also pertained to the availability of posters on FP and cue cards. We allocated one point to each type of visual aid and summed the points up to create an HF-based index which was averaged over all FP HFs to compute the mean number of items to support FP counseling in HFs in the LGA. Note that the $28 \%$ of all FP HFs with no visual aids and all LGAs that had no FP HFs were assigned the value of 0 on this indicator. The mean number of items to support FP counseling ranged from 0 to 2 .

Integration of FP with STI/MH services: This binary variable measured the presence/absence in the LGA of a FP HF that routinely provided services for STIs and counseled antenatal and postpartum care clients about FP. This information was derived from three questions:

(I) Are clients routinely treated for STls or are clients referred to another provider or location for STI treatment?

(2) Are antenatal care clients routinely counseled about FP?

(3) Are postpartum clients routinely counseled about FP?

Index of FP provider-client interaction: Unlike the other LGA-level measures, this variable was derived from the household survey. Current users of contraception were asked where they first obtained the current method and the type of provider they had spent the most time with when they first obtained the method. A subsequent question asked about various aspects of that interaction: "During that visit, did the provider you saw:

(a) Greet you?

(b) Respond to your questions/health concerns?

(c) Explain possible side effect or problems you might have with the method?

(d) Explain what to do about side effects?

(e) Tell you when to return for the next visit? (f) Treat you with respect?"

The value "I" was assigned to each aspect if it was reportedly performed by the provider, and " 0 ", otherwise, and an additive index created. This index captured interpersonal relations as well as information provided to clients that could enable informed choice and promote client satisfaction during service interactions. The additive index was averaged over all current users of contraception in the LGA to create a measure of the overall quality of provider-client interaction. The index ranged from 0 to 6.

Perceived need for more staff and emergency transport: These were two separate binary measures of the general service provision environment and 
were based on information collected from FP HWs about the problems they think should be addressed to improve their working situation and services: "What is the most important issue that you feel needs to be addressed to improve your work in family planning?" Only spontaneous responses were recorded.

State: State was a five-category variable indicating whether the LGA was based in the FCT or in the states of Bauchi, Kano, Lagos (reference group) or Nasarawa.

\section{Individual-level variables}

Control variables included age (as reported in continuous years); sex; highest level of school attended (none (reference group), primary, or secondary or higher); current employment status (unemployed or employed), religion (Christian or non-Christian), and type of place of residence (urban (reference group), semi-urban or rural). Marital status was divided into three categories: married (reference group), living together, and not in union. PCA was used to construct an index of household wealth based on the presence of particular amenities or items in the household. The items considered were: a refrigerator, electricity, piped water, flush toilet, a bicycle, a motorcycle, a car, television, a radio, and a telephone/cellular phone. Scree-plot inspection revealed a distinct one-factor solution. Therefore, the first component was used as the measurement of the household wealth index since it explained the major part of the common variances of all the ten components (44\%). Scale reliability (alpha coefficient) for the resultant index of household wealth was 0.820 and the $\mathrm{KMO}$ coefficient was 0.867 . From the predicted wealth index, households were grouped into terciles representing low (reference group), medium and high household wealth.

Each regression also controlled for the number of sources of information on FP to which the respondent was exposed. This variable was derived from the following question, which was administered to all respondents: "In the last 6 months have you heard or seen anything about family planning: a) On the radio? b) On television? c) In a newspaper or magazine? d) From a home visit by a community health worker? e) At the clinic? f) From a PMV? g) Anywhere else or from anyone else?" The value of one was assigned to each information source. Respondents' perception of community approval of FP was included in the contraceptive use regressions and was based on the question: "Do you think that a few, some, most or almost all the women/men in this community are in favor of the use of modern family planning?" and comprised three categories: None (reference group), a few, and some/most/all.

\section{Statistical Methods}

We used F-tests to examine differences between LGAs in the prevalence of knowledge and use of modern contraception by selected measures of the quality of the FP service delivery environment. For the multivariate analysis, we estimated multilevel logistic regression models using the generalized linear latent and mixed model command (GLLAMM) in Stata II.0 (Rabe-Hesketh and Skrondal, 2008). In the analysis, individuals were nested within the administrative boundaries of LGAs to detect associations of the FP service delivery environment with contraceptive outcomes. Multilevel modeling incorporated random effects at the individual and LGA-levels in the regression to model to account for unobserved factors.

Intra-class correlation coefficients (ICC) were used to evaluate how the outcomes of interest varied between LGAs and can be interpreted as the proportion of variation in the outcome that can be explained at the LGA-level. If a given measure of the FP service delivery environment was constant within each LGA, the only variation would be between LGAs, and the ICC would be close to I.0. In contrast, if most of the variation in a given outcome was explained by individual-level measures, the ICC would be close to 0 . For a two-level logistic random intercept model with a random intercept variance of $\sigma 2 \mu$, the intra-class correlation is:

$$
\rho=\left(\sigma_{\mu}^{2} /\left(\sigma_{\mu}^{2}+\pi^{2} / 3\right)\right)
$$

where, $\pi^{2} / 3=3.29$ and represents the level-I residual variance for a logit model.

We estimated odds ratios (ORs) and 95\% confidence intervals $(\mathrm{Cls})$ from regression statistics. To test for muticollinearity, we calculated variance inflation factors (VIFs) for explanatory variables included in each regression. The average VIF was 2.4 and the highest VIF was 4.5 (obtained for the mean number of contraceptive methods provided), signifying that a given independent variable was not highly correlated with the other independent variables in the regression of interest. We examined interaction terms between gender and selected variables, namely: (I) the presence in the LGA of a FP $\mathrm{HF}$ with all basic equipment for FP examination and (2) the quality of FP provider-client interaction in the LGA. We also examined interaction terms between type of place of residence and selected characteristics of the FP service delivery environment.

Of the 2218 male and 2250 females successfully interviewed, those with missing data on any of the variables of interest were excluded from the analysis, resulting in a sample size of $4 \mathrm{I} 24$ respondents for the http://aps.journals.ac,za 
regressions on knowledge of three or more modern methods of contraception of modern methods of FP, and $394 \mathrm{I}$ respondents for the regressions on current use of a modern method. It is to be noted that 357 respondents were excluded from the analysis because they were not completely interviewed or because their LGA of residence was not included in the HF sample, and that currently pregnant women were omitted from the regressions on current use of a modern method. The total number of LGAs included in the analysis was 45, with the number of respondents per LGA ranging from I 3 to 325 .

\section{Results}

\section{Sample Characteristics}

Table I provides the weighted socio-demographic characteristics of the sample as well as the characteristics of the FP service delivery environment in their LGAs of residence, by sex. Respondents were 32 years old on average. Slightly more than a third were employed, $40 \%$ were Christian, and close to half were married. The average respondent had been exposed to I.8 sources of information about FP and nearly one in four respondents felt that most women/men in the community disapproved of FP. The average respondent lived in an LGA in which FP HFs provided about 5.4 different methods of contraception and implemented at least 2 systems that supported quality service delivery. Nearly half of respondents resided in LGAs in which FP HWs perceived a need for more staff and $5 \%$ in LGAs where HWs perceived a need for emergency transportation.

Half of respondents lived in an LGA that had a FP $\mathrm{HF}$ with all basic resources for FP examination and in an LGA with a HF that provided FP in MH services. Significant gender differences in age, education, current employment, marital status, household wealth, and perceived community approval of FP were observed. In general, more males were educated to secondary or higher levels, employed, and not in union, and resided in rural areas, in Bauchi, and in the wealthiest households compared to females.

Table I: Characteristics of the sample, Nigeria 2009

\begin{tabular}{cccccc}
\multicolumn{2}{c}{ Male } & \multicolumn{2}{c}{ Female } & \multicolumn{2}{c}{ Total } \\
\hline \% or Mean & $\mathrm{N}$ & \% or Mean & $\mathrm{N}$ & \% or Mean & $\mathrm{N}$ \\
$(\mathrm{SE})$ & & $(\mathrm{SE})$ & & $(\mathrm{SE})$ & \\
& & & & $31.7(0.287)$ & 4124
\end{tabular}

Age ****

$33.6(0.434) \quad 2068$

$29.8(0.313) \quad 2056$

$31.7(0.287)$

Education **

$\begin{array}{lllllll}\text { None } & 23.4 & 508 & 28.9 & 662 & 28.5 & 1,174 \\ \text { Primary } & 29.9 & 630 & 30.5 & 632 & 30.6 & 1,262 \\ \text { Secondary/higher } & 46.7 & 930 & 40.6 & 758 & 40.9 & 1,688\end{array}$

Current employment $* * *$

Unemployed
Employed

1104

67.8

1447

62.6

50.2

964

32.2

579

37.4

Religion

$\begin{array}{lllllll}\text { Non-Christian } & 61.2 & 1275 & 58.8 & 1221 & 60.5 & 2,496 \\ \text { Christian } & 38.8 & 793 & 41.2 & 835 & 39.5 & 1,628\end{array}$

Marital status ****

Married

43.6 


594

3781

$\mathrm{I}, 493$

Household wealth **

$\begin{array}{lllllll}\text { Low } & 30.6 & 738 & 25.2 & 633 & 27.8 & \text { I,37। } \\ \text { Medium } & 32.3 & 636 & 38.7 & 733 & 35.5 & 1,369 \\ \text { High } & 37.1 & 692 & 36.1 & 690 & 36.6 & 1.382 \\ & 1.720(0.064) & 2068 & 1.791(0.064) & 2056 & 1.756 & 4124\end{array}$

No. of FP information sources

Perceived level of approval of FP

in community $* *$

None

25.2

551

19.3

446

22.2

997

Some/most/all

34.5

705

31.3

647

32.9

1352

40.4

812

49.5

963

44.9

1775

Type of place of residence ${ }^{*} *$

$\begin{array}{lllllll}\text { Urban } & 54.5 & 855 & 61.7 & 927 & 58.1 & 1782 \\ \text { Semi-urban } & 14.6 & 403 & 15.0 & 440 & 14.8 & 843 \\ \text { Rural } & 30.9 & 810 & 23.3 & 689 & 27.1 & 1499\end{array}$

Table I continued

\begin{tabular}{llllll}
\multicolumn{2}{c}{ Male } & \multicolumn{2}{c}{ Female } & \multicolumn{2}{c}{ Total } \\
\hline \% or Mean & $\mathrm{N}$ & \% or Mean & $\mathrm{N}$ & \% or Mean & $\mathrm{N}$
\end{tabular}

(SE)

(SE)

(SE)

\section{LGA-level variables}

Mean no. of FP methods

$5.226(0.283) \quad 2068$

5.665

2056

5.445

4124

provided

(0.299)

(0.278)

Mean no. of QA methods used

$2.828(0.186) \quad 2068$

2.791

2056

2.809

4124

by FP HFs

(0.179)

$(0.174)$

High FP supervision

57.5

2068

58. 1

2056

57.8

$4 \mid 24$

Mean no. of methods used by FP

$0.134(0.030) \quad 2068$

0.169

2056

0.151

4124

HFs to solicit client opinion

$(0.038)$

(0.032)

Index of HW training in FP

$-0.1072 \quad 2068$

$-0.050$

2056

$-0.078$

4124

$(0.197)$

(0.205)

$(0.191)$ 


\begin{tabular}{|c|c|c|c|c|c|c|}
\hline Availability of basic resources for & 55.7 & 2068 & 55.7 & 2056 & 55.7 & 4124 \\
\hline \multicolumn{7}{|l|}{ FP examination } \\
\hline Mean no. of items to support FP & $0.4 I I(0.045)$ & 2068 & 0.408 & 2056 & 0.410 & 4124 \\
\hline counseling & & & $(0.048)$ & & $(0.045)$ & \\
\hline Integration of FP with STI/MH & 68.0 & 2068 & 71.8 & 2056 & 69.9 & 4124 \\
\hline \multicolumn{7}{|l|}{ services } \\
\hline Index of FP provider-client & $2.707(0.158)$ & 2068 & 2.683 & 2056 & 2.695 & 4124 \\
\hline interaction & & & $(0.127)$ & & $(0.135)$ & \\
\hline Perceived need for more staff & 53.3 & 2068 & 51.0 & 2056 & 52.2 & 4124 \\
\hline Perceived need for emergency & 5.3 & 2068 & 4.1 & 2056 & 4.7 & 4124 \\
\hline \multicolumn{7}{|l|}{ transport } \\
\hline \multicolumn{7}{|l|}{ State ${ }^{* * * *}$} \\
\hline Lagos & 42.7 & 505 & 51.0 & 604 & 46.9 & 1109 \\
\hline Bauchi & 17.7 & 339 & 11.9 & 228 & 14.8 & 567 \\
\hline FCT & 4.3 & 297 & 5.0 & 352 & 4.6 & 649 \\
\hline Kano & 29.5 & 618 & 25.7 & 540 & 27.6 & 1158 \\
\hline Nasarawa & 5.9 & 309 & 6.4 & 332 & 6.1 & 641 \\
\hline
\end{tabular}

Data are weighted. Significance levels pertain to gender differences in background characteristics. *** $\mathrm{p}<.001 * * \mathrm{p}<.01 \quad * \mathrm{p}<.05$

\section{Knowledge of Modern Contraception}

As Table 2 shows, approximately two out of five respondents could spontaneously report three or more modern methods of contraception, with levels of knowledge being significantly higher among females than males $(46 \%$ versus $38 \% ; F(I, 4 I 76)=$ 17.0619; $\mathrm{p}<.001)$. Among both males and females, knowledge of modern contraception was lower in LGAs that had relatively low levels of (I) method choice potential; (2) QA methods used by FP nonPMVs; and (3) FP supervision; that routinely provided integrated $\mathrm{FP} / \mathrm{STI} / \mathrm{MH}$ services; and that had a perceived need for emergency transportation for patients. For example, only $34 \%$ of respondents could spontaneously recall three or more modern contraceptives in LGAs that ranked low in terms of the number of contraceptive methods provided compared to $47 \%$ in LGAs that ranked high on that indicator. These differentials were statistically significant at the one percent level.
Surprisingly, the proportion of males and females who knew three or more modern methods of contraception was higher in LGAs in which the average number of items to support counseling in FP HFs was less than one $(41 \%$ of males and $49 \%$ of females) than in other LGAs (35\% of males and $42 \%$ of females). This differential was statistically significant. Some sex-specific associations were found. The overall quality of provider-client interaction in the LGA and the availability of all basic resources for FP examination were significantly associated with the level of contraceptive knowledge among females but not among males. An expressed need for more staff in any of the FP HFs in the LGA was associated with significantly lower levels of contraceptive knowledge among males (35\% versus $43 \%$ if such a need was not mentioned) but not among females. 
Current Use of Modern Contraception

The modern contraceptive prevalence rate was low $(13 \%)$ and gender differences were not statistically significant (not shown). Among males and females, a high level of utilization of QA methods by FP nonPMVs, the LGA-level availability of a FP HF with all basic resources for FP examination, the presence of FP-integrated services and high quality of FP provider-client interaction were positively associated with the prevalence of modern contraceptive use among both males and females. Perceived shortages of staff and emergency transportation by FP providers in an LGA - a reflection of service delivery needs-were associated with lower rates of contraceptive use among both male and female residents. For example, only $9 \%$ of women reported current use of a modern method of contraception in LGAs with a perceived need for staff compared to $15 \%$ in LGAs with no such perceived need. The positive association between the index of supervision and the contraceptive prevalence rate was statistically significant only among males. Although statistically significant, some of the differences in prevalence rates were small when comparing LGAs, which could be partly due to the low overall prevalence of contraceptive

use.

Table 2: Percent of respondents who knew three or more modern methods of contraception and were currently using a modern method by selected characteristics of the FP service environment in the LGA of residence and sex, Nigeria 2009

Knowledge Current Use

\begin{tabular}{|c|c|c|c|c|c|c|}
\hline LGA Characteristics & Male & Female & Total & Male & Female $^{a}$ & Total \\
\hline Mean no. of FP methods provided & **** & **** & **** & **** & Ns & **** \\
\hline Below Median & 30.8 & 38.7 & 34.3 & 10.2 & 10.0 & 10.1 \\
\hline At/above Median & 44.6 & 49.8 & 47.4 & 16.7 & 12.9 & 14.8 \\
\hline Mean no. of QA methods used by FP HFs & $* * *$ & $* * *$ & $* * *$ & **** & $* * *$ & **** \\
\hline Below Median & 31.8 & 39.3 & 35.5 & 9.9 & 8.1 & 9.0 \\
\hline At/above Median & 42.8 & 49.9 & 46.4 & 16.5 & 14.3 & 15.4 \\
\hline FP supervision index & 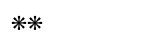 & $* * *$ & $* * *$ & * & Ns & $*$ \\
\hline Below Median & 34.8 & 39.6 & 37.2 & 11.3 & 11.2 & 11.3 \\
\hline At/above Median & 41.2 & 50.2 & 45.7 & 15.7 & 12.7 & 14.1 \\
\hline
\end{tabular}

Mean no. of methods used by FP HFs to

solicit client opinion

0

।

2

Index of provider training in FP

Below Median

At/above Median

Availability of basic resources for FP

$\begin{array}{llcccc}\text { ns } & \text { ns } & \text { ns } & \text { ns } & \text { Ns } & \text { ns } \\ 38.1 & 47.0 & 42.4 & 13.8 & 11.9 & 12.9 \\ 41.0 & 39.6 & 40.3 & 14.6 & 11.7 & 13.1 \\ 39.1 & 35.7 & 37.3 & 8.7 & 18.5 & 14.0 \\ * * * & \mathrm{~ns} & * * & \mathrm{~ns} & * & * \\ 42.0 & 46.7 & 44.4 & 13.0 & 10.2 & 11.7 \\ 33.8 & 44.7 & 39.3 & 14.9 & 13.9 & 14.5\end{array}$




\begin{tabular}{|c|c|c|c|c|c|c|}
\hline \multirow[b]{2}{*}{ LGA Characteristics } & \multicolumn{3}{|c|}{ Knowledge } & \multicolumn{3}{|c|}{ Current Use } \\
\hline & Male & Female & Total & Male & Female $^{a}$ & Total \\
\hline No & 36.4 & 42.8 & 39.6 & 10.8 & 9.3 & 10.1 \\
\hline Yes & 40.1 & 48.1 & 44.1 & 16.3 & 13.9 & 15.1 \\
\hline Items to support FP counseling & ** & 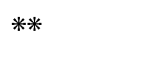 & ** & * & Ns & * \\
\hline 0 & $4 I .1$ & 48.5 & 44.8 & 15.6 & 12.3 & 14.6 \\
\hline 1 & 34.6 & 41.8 & 38.2 & 11.3 & 11.3 & 11.3 \\
\hline Integration of $\mathrm{FP}$ with $\mathrm{STI} / \mathrm{MH}$ services & $*$ & * & **** & **** & *** & **** \\
\hline No & 34.8 & 41.2 & 37.8 & 7.7 & 8.5 & 8.0 \\
\hline Yes & 40.1 & 47.6 & 44.0 & 16.7 & 13.2 & 15.0 \\
\hline
\end{tabular}

Table 2 Continued

\begin{tabular}{|c|c|c|c|c|c|c|}
\hline \multirow[b]{2}{*}{ LGA Characteristics } & \multicolumn{3}{|c|}{ Knowledge } & \multicolumn{3}{|c|}{ Current Use } \\
\hline & Male & Female & Total & Male & Female $^{\mathbf{a}}$ & Total \\
\hline Index of FP provider-client interaction & ns & ***** & ***** & * & **⿻丷木 & **** \\
\hline Low & 36.4 & 40.4 & 38.3 & 11.2 & 9.0 & 10.2 \\
\hline High & 40.1 & 49.8 & 45.1 & 16.0 & 14.0 & 15.0 \\
\hline Perceived need for more staff & ** & ns & ns & $* * *$ & ** & *** \\
\hline No & 42.7 & 44.8 & 43.4 & 18.2 & 14.6 & 16.5 \\
\hline Yes & 34.7 & 46.7 & 40.6 & 10.0 & 9.2 & 9.6 \\
\hline Perceived need for emergency & **** & * & **** & * & **⿻丷木 & **** \\
\hline \multicolumn{7}{|l|}{ transport } \\
\hline No & 39.3 & 46.3 & 42.8 & 14.2 & 12.2 & 13.3 \\
\hline Yes & 23.4 & 33.5 & 27.8 & 7.3 & 3.4 & 5.7 \\
\hline Total & 38.4 & 45.8 & 42.1 & 13.8 & 11.9 & 12.9 \\
\hline $\mathrm{N}$ & 2068 & 2056 & 4124 & 2068 & 1873 & 3941 \\
\hline
\end{tabular}

Data are weighted

a Excludes women who are currently pregnant or have never had sex

ns Not significant

*** $\mathrm{p}<.001 \quad$ *⿻丷木 $\mathrm{p}<.01 \quad$ * $\mathrm{p}<.05$

Multivariate Results

Table 3 presents the multivariate results. The average number of contraceptive methods provided and the average number of $\mathrm{QA}$ systems used to support service delivery in FP HFs in respondents' LGA of residence were positively associated with the odds of knowing three or more modern methods of FP, with the odds increasing by $12 \%$ with each additional FP method provided in the average FP clinic in the LGA $(\mathrm{OR}=1.120 ; 95 \% \mathrm{Cl}=1.029,1.220 ; \mathrm{p}=0.009)$. The higher was the number of $\mathrm{QA}$ methods used by 
FP HFs in an LGA, the greater was the likelihood that people living in the LGA knew three or more modern methods of contraception. Contrary to expectations, methods as compared to those who did not. This association was significant at the one percent level.

Three measures of the HF readiness and the quality of the FP service delivery environment were significantly associated with the odds of currently using a modern method of contraception. Respondents residing in LGAs with a perceived need for more staff among FP HWs had lower odds than other respondents of current modern contraceptive use $(\mathrm{OR}=0.627 ; 95 \% \mathrm{Cl}=0.45 \mathrm{I}, 0.87 \mathrm{I} ; \mathrm{p}=$ 0.005). The odds of currently using a modern method of contraception was significantly higher in LGAs with higher indices of FP HW training (OR = I. $187 ; 95 \% \mathrm{Cl}=1.092, \mathrm{I} .290 ; \mathrm{p}=0.00 \mathrm{I})$ and FP provider-client interaction $(\mathrm{OR}=1.298 ; 95 \% \mathrm{Cl}=$ I.I05, I.525; $p=0.001)$.

The individual-level results were worthy of note. The odds of knowing three or more modern methods of contraception were significantly higher for respondents who were older, educated, employed, Christian and had been exposed to more sources of FP information in the past six months, and were significantly lower in Bauchi than in Lagos State and among rural as compared to urban residents. Although respondents living in households with medium and high compared to low levels of wealth had significantly higher odds of knowing three or more modern methods of contraception, household wealth was not associated with the odds of current use of a modern method of contraception. For the models examining contraceptive use, the odds of current use of a modern method were significantly higher among respondents who were educated, employed, were exposed to more information sources, and perceived that few or some/most/all women or men in their community (as opposed to none) approved of FP. The odds of current use of a modern method were significantly lower in the states of Bauchi, Kano, and Nasarawa as compared to Lagos and surprisingly higher in rural as compared to urban areas.

\section{Random Effects}

To assess whether there were significant differences between LGAs in contraceptive outcomes we first respondents residing in LGAs in which FP counseling was integrated in STI/MH services had significantly lower odds of knowing three or more modern estimated a model with just a multilevel constant term, the LGA-specific random effect and no explanatory variables (not shown). The results from the null logit model yielded a level-2 (between-LGA) variance of 1.475 (standard error $(\mathrm{SE})=-0.365$ ) for knowledge of three or more modern methods and 2.48I ( $\mathrm{SE}=-0.742$ ) for current use of a modern method of contraception. We calculated an approximate Wald statistic for each outcome (Wald statistic $\left.=(\text { estimated level-2 variance } / \mathrm{SE})^{2}\right)$ and tested it in a chi-squared distribution with one degree of freedom. The resulting $p$-values were halved because the alternative hypothesis is one sided given that variances are by definition non-negative (i.e., HA: $\sigma^{2}{ }_{\mu}>0$ ). Our calculations yielded a Wald statistic of 16.3 for contraceptive knowledge, and II.2 for current use of a modern method, and $p<.001$ for both outcomes. Thus it can be concluded that difference between LGAs were highly significant for both outcomes. The significance of the random intercepts implied that contraceptive knowledge and decisions were determined by factors not captured by the observed covariates. Important unmeasured factors could have included variations in cultural beliefs surrounding contraceptive use and factors underlying the placement of FP services.

The relative importance of individual-level and LGAlevel variables in accounting for variation in contraceptive outcomes was estimated by calculating the ratio of the LGA-level variance to the total variance (the intra-class correlation), a measure of the degree to which contraceptive outcomes were clustered at the LGA-level. Using the estimates from the null model, we obtained an intra-LGA correlation of 0.310 for knowledge of three or more modern methods, and 0.430 for current use of a modern method of contraception. These estimates implied that even though more than half the variation in contraceptive outcomes is explained by individuallevel characteristics, there is a considerable proportion of the explained variance that is attributable to LGA-level measures of the FP service delivery 
Table 3: Multilevel logit regressions of knowledge or three or more modern methods and current use of a modern method, Nigeria 2009

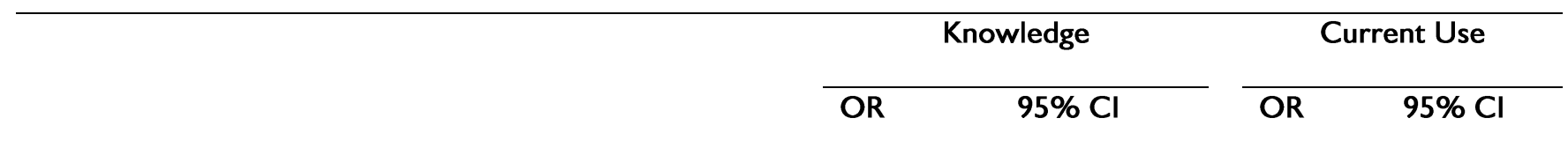

Individual-level variables

(0.992,

Age

$1.018 * * * \quad(1.010,1.026) \quad 1.004 \quad 1.017)$

Sex

Male

1.00

1.000

(0.720,

Female

I.4I3**** $\quad(1.202,1.661) \quad 0.905$

I. 138)

Education

None

1.000

1.000

(I.)16,

Primary

$1.959 * *$

(I.566, 2.45I)

$|.69| *$

2.562)

(I. I73,

Secondary/higher

$2.519 *$

$(1.995,3.179)$

I.772** 2.677 )

Current employment

Unemployed

1.000

1.000

(1.367,

Employed

I.296** $\quad(1.098,1.529)$

I.733***

2. 198)

Religion

Non-Christian

1.000

1.000

(0.955,

Christian

I.548***

$(1.290,1.857)$

1.218

I.554)

Marital status

Married

1.000

1.000

(0.713

Living together

1.057

$(0.843,1.324)$

0.982

I.354)

(0.89),

Not in union

$0.772 * *$

$(0.638,0.934)$

1.172

I.543) 
Household wealth

\begin{tabular}{llllll} 
Low & 1.000 & \multicolumn{3}{c}{1.000} \\
Medium & & & & $(0.947$, \\
& $1.428 * *$ & $(1.128,1.806)$ & 1.392 & $2.046)$ \\
High & & & & $(0.960$, \\
& $1.451 * *$ & $(1.123,1.876)$ & 1.441 & $2.164)$ \\
& & & & $(1.078$, \\
& & & & $1.147^{* * * *}$ & $1.221)$
\end{tabular}

No. of FP information sources

Perceived level of approval of FP among women in community

None

1.000

$(1.317$

Few

$2.106 * * 3.367)$

$(1.898$,

Some/most/all

$2.988 * * * \quad 4.706)$

Table 3 continued

\begin{tabular}{|c|c|c|c|c|}
\hline 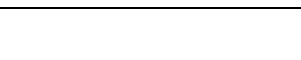 & \multicolumn{2}{|c|}{ Knowledge } & \multicolumn{2}{|c|}{ Current Use } \\
\hline & OR & $95 \% \mathrm{Cl}$ & OR & $95 \% \mathrm{Cl}$ \\
\hline \multicolumn{5}{|c|}{ Type of place of residence } \\
\hline Urban & 1.000 & 1.000 & 1.000 & 1.000 \\
\hline Semi-urban & 0.895 & $(0.684,1.169)$ & 0.965 & I.364) \\
\hline Rural & $0.683 *$ & $(0.489,0.955)$ & $1.182 *$ & 1.973) \\
\hline
\end{tabular}

LGA-level variables

Mean no. of FP methods provided

$\begin{array}{llll}1.12 I^{* *} \quad(1.029,1.221) & 0.999 & 1.085)\end{array}$

Mean no. of QA systems used by FP HFs

$\begin{array}{llll}1.142 * \quad(1.010,1.290) & 1.039 & 1.162)\end{array}$

(0.59),

High FP supervision

1.165

$(0.722, \mid .88 I)$

0.961

I.563) 
African Population Studies Vol 3I, No I,(Supp.), 2017

Index of provider training in FP

0.965

$(0.87 I, I .069)$

I. $187 * * * \quad \mid .290)$

$(0.691$,

Mean no. of methods used by FP HFs to solicit client opinion

0.739

$(0.487,1.121)$

0.984

1.402)

$(0.643$

Availability of basic resources for FP examination

0.931

$(0.615,1.409)$

0.998

I.549)

$(0.531$,

Items to support FP counseling

0.766

$(0.499,1.177)$

0.758

I.083)

$(0.306$

Integration of FP with STI/MH services

$0.472 * *$

$(0.269,0.826)$

0.699

I.597)

(1.105,

Index of FP provider-client interaction

$1.298^{* * *} \quad 1.525$

$(0.451$,

Perceived need for more staff

1.223

$(0.839,1.784)$

$\left.0.627^{* *} \quad 0.87 \mathrm{I}\right)$

(0.398,

Perceived need for emergency transport

0.835

$(0.370,1.88 I)$

0.799

1.603)

State

Lagos

1.000

1.000

$(0.063$

Bauchi

$0.404 *$

$(0.202,0.810)$

$\left.0.153^{* * *} \quad 0.374\right)$

(0.370,

FCT

1.165

$(0.596,2.274)$

0.609* 1.001 )

(0.048,

Kano

$0.565 *$

$(0.341,0.934)$

0.097

$0.194)$

(0.247,

Nasarawa

0.961

$(0.508,1.818)$

$0.472 * \quad 0.903)$

Constant (SE)

$-2.776 * * * *(0.415)$

$-4.103(0.572)$

LGA Random Part

Variance (covariance)

$0.179(0.062)$

$6.615 e-19(2.527 \mathrm{e}-10)$

No. of respondents

4124

3941 


\section{Interactions}

In Table 4, we present the results of regression models with interaction terms between type of place of residence and the following LGA-level variables: mean number of FP methods provided, mean number of QA methods used by FP HFs index of provider training in FP, high FP supervision, and the index of FP provider-client interaction. The interaction terms were added to the models shown in Table 3. The results demonstrated that the odds of knowing three or more modern methods of contraception were 19\% higher among rural residents in LGAs that offered more contraceptive methods and $32 \%$ and $23 \%$ higher among semiurban and rural residents, respectively, in LGAs that used more $\mathrm{QA}$ methods than among urban residents in those LGAs.

By comparison, the odds of knowing three or more modern methods were lower among rural residents in LGAs with higher indices of provider training in FP and among semi-urban residents in LGAs with high FP supervision of FP non-PMVs compared with urban residents in those LGAs. In LGAs that provided a higher number of FP methods, the odds of currently using a modern method were lower among respondents in semi-urban areas as compared with those residing in urban areas. In LGAs with a higher number of QA methods used by FP HFs and in LGAs with higher indices of providerclient interaction, there were $52 \%$ and $56 \%$ higher odds of currently using a modern method in semiurban areas than in urban areas.

We also tested for interactions between gender and (a) the index of FP provider-client interaction and (b) the availability of basic resources for FP examination. The results (not shown) indicated that the odds of currently using a modern method among respondents residing in LGAs with higher indices of FP provider-client interaction were significantly higher for women than for men (OR $=1.294 ; 95 \%$ $\mathrm{Cl}=1.063,1.575 ; \mathrm{P}=0.010)$.

Table 4: Multilevel logit regression of contraceptive outcomes with selected type-of-place-of-residence interactions, Nigeria 2009

\begin{tabular}{lllll}
\hline & \multicolumn{2}{c}{ Knowledge } & & \multicolumn{2}{c}{ Current Use } \\
\cline { 2 - 3 } & OR $\quad 95 \% \mathrm{Cl}$ & & OR $95 \% \mathrm{Cl}$ \\
\hline
\end{tabular}

Individual-level Variables

Type of place of residence

Urban

Semi-urban

Rural

\section{LGA-level Variables}

Mean no. of FP methods provided

Mean no. of QA methods used by FP HFs

Index of provider training in FP

High FP supervision

Index of FP provider-client interaction

$$
1.000
$$

0.603

$0.217^{* * * *}$

$(0.301,1.207)$

0.193

$(0.097,0.487)$

0.311

$(0.030, I .267)$

$(0.047,2.066)$

\section{Interaction-terms}

$\begin{array}{llll}1.054 & (0.972,1.143) & 1.008 & (0.923,1.102) \\ 0.983 & (0.859,1.125) & 1.060 & (0.918,1.224) \\ 1.037 & (0.931,1.155) & 1.225^{* * *} & (1.106,1.357) \\ 1.940 * & (1.095,3.437) & 0.616 & (0.303,1.250) \\ & & 1.141 & (0.861,1.513)\end{array}$




\begin{tabular}{|c|c|c|c|c|}
\hline Semi-urban * Mean no. of FP methods provided & 1.046 & $(0.955,1.144)$ & $0.816^{*}$ & $(0.693,0.961)$ \\
\hline Rural * Mean no. of FP methods provided & $1.190 * * *$ & $(1.079,1.313)$ & 1.133 & $(0.966,1.329)$ \\
\hline Semi-urban * Index of provider training in FP & 0.985 & $(0.817,1.186)$ & 0.873 & $(0.674,1.132)$ \\
\hline Rural * Index of provider training in FP & $0.745^{* *}$ & $(0.616,0.901)$ & 0.970 & $(0.751,1.253)$ \\
\hline Semi-urban * Mean no. of QA methods used by FP HFs & $1.320 * *$ & $(1.106,1.574)$ & $1.517^{*}$ & $(1.084,2.122)$ \\
\hline Rural * Mean no. of QA methods used by FP HFs & $1.227^{*}$ & $(1.034,1.455)$ & 0.891 & $(0.677,1.174)$ \\
\hline Semi-urban * High FP supervision & $0.442^{*}$ & $(0.215,0.906)$ & 1.563 & $(0.570,4.283)$ \\
\hline Rural $*$ High FP supervision & 0.779 & $(0.373,1.627)$ & 1.381 & $(0.459,4.153)$ \\
\hline Semi-urban * Index of FP provider-client interaction & & & $1.557^{*}$ & $(1.044,2.322)$ \\
\hline Rural * Index of FP provider-client interaction & & & 1.218 & $(0.888, I .67 I)$ \\
\hline Constant (SE) & \multicolumn{2}{|c|}{$-2.305 * * * \quad(0.402)$} & \multicolumn{2}{|c|}{$-3.630(0.700)$} \\
\hline \multicolumn{5}{|l|}{ LGA Random Part } \\
\hline Variance (covariance) & \multicolumn{2}{|c|}{$0.096(0.050)$} & \multicolumn{2}{|c|}{$9.986 \mathrm{e}-21(1.444 \mathrm{e}-1 \mathrm{I})$} \\
\hline No. of respondents & \multicolumn{2}{|c|}{$4 \mid 24$} & \multicolumn{2}{|c|}{3941} \\
\hline No of LGAs & \multicolumn{2}{|c|}{45} & \multicolumn{2}{|r|}{45} \\
\hline Log likelihood & \multicolumn{2}{|c|}{-2191.849} & \multicolumn{2}{|c|}{-1107.1122} \\
\hline
\end{tabular}

\section{Discussion}

This study filled an important gap in the literature on what is known about the association between Nigeria's FP supply environment and knowledge and use of modern methods of contraception. The results of our analysis showed that the mean number of FP methods provided and the number of QA methods used by FP HFs in the LGA were significantly associated with an increased likelihood of knowing three or more modern methods. We also found that residents of LGAs characterized by higher indices of provider training in FP interaction had higher odds of current use of a modern method of contraception. Evidence suggests that HW training improves providers' knowledge and the quality of FP services provided to clients (Jain et al., 20I2), improves client satisfaction (Chin-Quee et al., 20l6), and increases clients' likelihood to return for follow-up visits (Costello et al., 200I, RamaRao and Mohanam, 2003).

Not surprisingly, the results also showed that the odds of modern contraceptive use were higher in LGAs with higher indices of FP provider-client interaction than in other LGAs, and that this association was significantly stronger in semi-urban than in urban areas. A comparison of these findings with previous studies that have examined the influence of HF readiness and the quality of care on contraceptive behavior was limited by the variety of indicators that have been used to define these concepts, the different methodologies used for data collection, and the different outcomes examined. Bearing these differences in mind, our findings are in line with those of other authors who have examined how individual and community perceptions of the quality of available FP services are related to contraceptive adoption. For example, Hong et al. (2006) found that the quality of services related to counseling and the exam room had strong positive associations with IUD use in Egypt (see Koenig et al. (1997)).

The analysis also demonstrated that the odds of current use of a modern method of contraception were lower in LGAs with perceived shortages of medical staff than in LGAs without these shortages. While perceived shortages of medical staff are indicative of human resources for the provision of health services, they could also be a reflection of 
limited funding, payment schemes and incentives; poor geographic access; poor working conditions which make it difficult to retain HWs; long waiting times; unnecessary medical policies and practices which increase HW burden; or the sex and ethnic composition of providers which could affect psychosocial aspects of the utilization of FP services. Additional data are necessary, therefore, to better understand these findings.

\section{Limitations}

Although this study has highlighted the importance of several components of health service delivery for contraceptive knowledge and use, the analysis has some limitations. Due to lack of data, we were unable to measure directly two key element of Bruce's (1990) framework: technical competence (which encompasses providers' clinical techniques and observance of protocols and asepsis in clinical conditions) and mechanisms for ensuring continuity and follow-up (which could include both mass media and client-based follow-up mechanisms, including return appointments and home visits to clients). Chart abstraction, clinical vignettes, and direct observation and recording of visits are commonly used in low-income countries to measure the process aspects of quality. However, these methodologies were not incorporated into the COMPASS surveys. While our index of FP provider-client interaction incorporated aspect of interpersonal relations as well as information given to clients during FP interactions, we were unable to measure the level of empathy, confidentiality, privacy; and sensitivity of providers to clients' needs.

Another limitation of the analysis stemmed from the cross-sectional nature of the data, which made it difficult to establish causality. Endogeneity was of concern because decisions about the placement of services are often not random. If FP HFs are placed and higher contraceptive technology provided in LGAs where demand for contraceptives is high, failure to consider this factor could lead to an overstatement of the effects of the FP service delivery environment. If FP services are integrated in $\mathrm{MH}$ services in LGAs with low contraceptive prevalence, the effects of service characteristics could be understated (Steele et al., 1999). It may also happen that users of modern contraception may selectively migrate to LGAs with higher levels of facility readiness and higher quality services. If so, the observed correlations between the LGA service environment and contraceptive outcomes may be a reflection of unmeasured processes by which individuals sort themselves into different LGAs (see Sampson (2003)).
The literature suggests three ways of dealing with endogeneity: (I) actually measuring crucial omitted variables; (2) applying multilevel instrumental variables estimation techniques; (3) using repeated measures longitudinal fixed effects models (Subramian et al., 2003). Some studies have also addressed issues of causality by implementing experimental and quasi-experimental designs. However, none of these methodologies were within the scope of the present study due, in part, to the difficulty of finding instruments and to the fact that the facility and household surveys did not implement a panel design. Further work will be necessary, therefore, to clarify the causal mechanisms by which contraceptive outcomes are influenced by FP service delivery factors.

One methodological issue in the present study was defining the relevant higher-level units to be included in the multilevel analysis. Many studies of contextual and community effects on health outcomes in sub-Saharan have utilized the EA or census tract to define higher-level units. Recognizing that individuals may go several communities away in search of better health services, the sampling frame for the 2009 facility survey was based only on HFs (including all public and private primary HFs) serving the populations interviewed in the baseline, midline, and end-of-project household surveys. Hence, the HF survey included some facilities that were located outside the EAs selected for the household survey, thereby violating the inherent assumption in our multilevel analysis that people living in the same geographic area shared similar FP services. The use of a higher level of aggregation than the EA to link the service environment to contraceptive outcomes may not have completely addressed this problem. Six of the 5I LGAs targeted by the COMPASS project were omitted from the present study as they were not canvassed in the HF survey. In some LGAs the number of HFs surveyed was small, raising questions as to the extent to which these service delivery points indicated the level of readiness of the LGA to offer FP services. These issues can only be addressed through a HF census but the costs of implementing such a study could be prohibitive.

Despite these methodological constraints, we believe that our FP service environment measures were more meaningful at the higher level of aggregation (i.e., the LGA) than at the lower level (i.e., the EA). As community-specific estimates depend on the sample size in specific communities, defining our level-2 units in terms of the LGA also ensured a higher number of individuals in each level-2 unit, an issue that is related to the precise power of calculations within multilevel models. Note also that the HF and household surveys were conducted in the 
5I LGAs that were targeted at the start of the COMPASS project; hence the results of our analysis were not representative of all HFs and households in the FCT and in the states of Bauchi, Lagos, Kano, and Nasarawa.

\section{Conclusion}

The findings call for interventions that target specific elements of FP service-delivery. First, expanding contraceptive choice by introducing more methods to existing FP programs in LGAs that offered fewer contraceptive methods could expand individual's awareness of how different methods could be used to satisfy diverse family needs. Second, training health care providers in specific elements of FP service delivery, including FP counseling and technical competence can facilitate clients' decision making to adopt modern methods of contraception. The significant negative associations with contraceptive use of HW identification of shortages of staff in FP clinics point to the importance of having providers in an LGA evaluate their own services to identify constraints to service delivery. The use of provider and HF self-assessment tools for improving the quality of care, such as the Client-Oriented ProviderEfficient tool (Engenderhealth, 2003), could be encouraged while recognizing that possible solutions to staff shortages and other problems may require sizeable investment of resources, close collaboration between healthcare providers and management, and political commitment to implement solutions.

\section{Acknowledgements}

MEASURE Evaluation Phase III was funded by the U.S. Agency for International Development (USAID) through Cooperative Agreement GHA-A-00-0800003-00 and was implemented by the Carolina Population Center at the University of North Carolina at Chapel Hill in partnership with Futures Group, ICF Macro, John Snow, Inc., Management Sciences for Health, and Tulane University. The PRH Associated Award was funded through Associate Award GPO-A-00-09-0003-00. The authors' views expressed in this publication do not necessarily reflect the views of USAID or the United States government. We are grateful to Professor Adewuyi for his guidance and contributions at all stages of survey implementation.

\section{References}

ALI, M. M. 200I. Quality of care and contraceptive pill discontinuation in rural Egypt. J Biosoc Sci, 33, I6I-72.

ARENDS-KUENNING, M. \& KESSY, F. L. 2007. The impact of demand factors, quality of care and access to facilities on contraceptive use in Tanzania. J Biosoc Sci, 39, I-26.

ASEKUN-OLARINMOYE, E., ADEBIMPE, W., BAMIDELE, J., ODU, O., ASEKUNOLARINMOYE, I. \& OJOFEITIMI, E. 2013. Barriers to use of modern contraceptives among women in an inner city area of Osogbo metropolis, Osun state, Nigeria. Int J Womens Health, 5, 647-55.

AUSTIN, A. 20I5. Unmet contraceptive need among married Nigerian women: an examination of trends and drivers. Contraception, 9I, 3I-8.

BAUMGARTNER, J. N., OTIENO-MASABA, R., WEAVER, M. A., GREY, T. W. \& REYNOLDS, H. W. 2012. Service delivery characteristics associated with contraceptive use among youth clients in integrated voluntary counseling and HIV testing clinics in Kenya. AIDS Care, 24, I290-30I.

BRUCE, J. 1990. Fundamental elements of the quality of care: a simple framework. Stud Fam Plann, 2I, 6I-9I.

CAMPBELL, O. M., BENOVA, L., MACLEOD, D., GOODMAN, C., FOOTMAN, K., PEREIRA, A. L. \& LYNCH, C. A. 2015. Who, what, where: an analysis of private sector family planning provision in 57 low- and middle-income countries. Trop Med Int Health, 20, I639-56.

CHARYEVA, Z., OGUNTUNDE, O., OROBATON, N., OTOLORIN, E., INUWA, F., ALALADE, O., ABEGUNDE, D. \& DANLADI, S. 20I5. Task shifting provision of contraceptive implants to community health extension workers: results of operations research in Northern Nigeria. Glob Health Sci Pract, 3, 382-94.

CHIN-QUEE, D., MUGENI, C., NKUNDA, D., UWIZEYE, M. R., STOCKTON, L. L. \& WESSON, J. 2016. Balancing workload, motivation and job satisfaction in Rwanda: assessing the effect of adding family planning service provision to community health worker duties. Reprod Health, 13, 2.

COSTELLO, M., LACUESTA, M., RAMARAO, S. \& JAIN, A. 200I. A client-centered approach to family planning: the Davao project. Stud Fam Plann, 32, 302-I4.

DO, M. P. \& KOENIG, M. A. 2007. Effect of family planning services on modern contraceptive method continuation in Vietnam. J Biosoc Sci, 39, 20I-20.

ENGENDERHEALTH 2003. COPE handbook: a process for improving quality in health services New York, Engenderhealth.

HONG, R., MONTANA, L. \& MISHRA, V. 2006. Family planning services quality as a determinant of use of IUD in Egypt. BMC Health Serv Res, 6, 79. 
JAIN, A. K. 1989. Fertility reduction and the quality of family planning services. Stud Fam Plann, 20, I- I6.

JAIN, A. K., RAMARAO, S., KIM, J. \& COSTELLO, M. 2012. Evaluation of an intervention to improve quality of care in family planning programme in the Philippines. J Biosoc Sci, 44, 27-4I.

KOENIG, M. A., HOSSAIN, M. B. \& WHITTAKER, M. 1997. The influence of quality of care upon contraceptive use in rural Bangladesh. Stud Fam Plann, 28, 278-89.

LEVY, J. K., CURTIS, S., ZIMMER, C. \& SPEIZER, I. S. 2014. Assessing gaps and poverty-related inequalities in the public and private sector family planning supply environment of urban Nigeria. J Urban Health, 9I, I86-210.

MAGNANI, R. J., HOTCHKISS, D. R., FLORENCE, C. S. \& SHAFER, L. A. 1999. The impact of the family planning supply environment on contraceptive intentions and use in Morocco. Stud Fam Plann, 30, 120-32.

NATIONAL POPULATION COMMISSION \& HEALTH POLICY PROJECT 20I5. Nigeria's 2004 National Policy on Population for Sustainable Development: Implementation Assessment Report, Washington, DC, Washington, DC: Futures Project. .

NATIONAL POPULATION COMMISSION \& ICF INTERNATIONAL 20I4. Nigeria Demographic and Health Survey 2013. Abuja, Nigeria, and Rockville, Maryland, USA: NPC and ICF International.

RABE-HESKETH, S. \& SKRONDAL, A. 2008. Multilevel and Longitudinal Modeling Using Stata, College Station, TX, Stata Press.

RAMARAO, S. \& MOHANAM, R. 2003. The quality of family planning programs: concepts, measurements, interventions, and effects. Stud Fam Plann, 34, 227-48.

ROSS, J. \& STOVER, J. 2013. Use of modern contraception increases when more methods become available: analysis of evidence from 19822009. Glob Health Sci Pract, I, 203-I 2.
SAMPSON, R. J. 2003. Neighborhood-level context and health: lessons from sociology. In: KAWACHI, I. \& BERKMAN, L. F. (eds.) Neighborhoods and Health. New York: Oxford University Press.

STEELE, F., CURTIS, S. L. \& CHOE, M. 1999. The impact of family planning service provision on contraceptive-use dynamics in Morocco. Stud Fam Plann, 30, 28-42.

SUBRAMIAN, S. V., JONES, K. \& DUNCAN, C. 2003. Multilevel methods for public health research. In: KAWACHI, I. \& BERKMAN, L. F. (eds.) Neighborhoods and Health. New York: Oxford University Press.

TESSEMA, G. A., LAURENCE, C. O., MAHMOOD, M. A. \& GOMERSALL, J. S. 2016. Factors determining quality of care in family planning services in Africa: a systematic review protocol. JBI Database System Rev Implement Rep, I4, I0314.

TSUI, A. O., CASTERLINE, J., SINGH, S., BANKOLE, A., MOORE, A. M., OMIDEYI, A. K., PALOMINO, N., SATHAR, Z., JUAREZ, F. \& SHELLENBERG, K. M. 20I I. Managing unplanned pregnancies in five countries: perspectives on contraception and abortion decisions. Glob Public Health, 6 Suppl I, SI-24.

TUMLINSON, K., PENCE, B. W., CURTIS, S. L., MARSHALL, S. W. \& SPEIZER, I. S. 20I5. Quality of Care and Contraceptive Use in Urban Kenya. Int Perspect Sex Reprod Health, 4I, 69-79.

UNEKE, C. J., NDUKWE, C. D., EZEOHA, A. A., UROCHUKWU, H. C. \& EZEONU, C. T. 2014. Improving maternal and child healthcare programme using community-participatory interventions in Ebonyi State Nigeria. Int J Health Policy Manag, 3, 283-7.

UNITED NATIONS 20I5. World Population Prospects, the 2015 Revision, New York, United Nations Population Division, Department of Economic and Social Affairs. 